\title{
Middle East and North African Health Informatics Association (MENAHIA):
}

\section{Building Sustainable Collaboration}

Najeeb Al-Shorbaji ${ }^{a}$, Mowafa Househ ${ }^{b}$, Adel Taweel', Abdullah Alanizib ${ }^{b}$ Bennani Othmani Mohammed , Haitham Abaza ${ }^{\mathrm{e}}$, Hala Bawadif, Hamayon Rasuly ${ }^{9}$, Khalid Alyafei ${ }^{\mathrm{h}}$, Luis Fernandez-Luquei, Mohamed Shoumani, Osama El-Hassank, Rada Hussein', Riyad Alshammarim, Salah Mandil", Sarah Shoumani, Shahrad Taheri ${ }^{\circ}$, Tamer Emarai, Wasmiya Dalhem ${ }^{p}$, Zaid Al-Hamdan ${ }^{q}$, Zineb Serhier ${ }^{r}$

" ellealth and Knowledge Management Consultant, Retired Director of Knowledge, Ethics and Research Department, WHO/HQ, Amman, Jordan

b Department of Health Informatics, College of Public Health and Health Informatics, King Saud Bin Abdulaziz University for Health Sciences, Ministry of National Guard-Health Affairs, Riyadh, Saudi Arabia c Department of Computer Science, BirZeit University, Ramallah, Palestine

d Head of Medical Informatics Unit, 20 August Hospital, Ibn Rochd University Hospital, Casablanca, Head of Casablanca Medical Informatics Laboratory, Medical Faculty of Casablanca, Morocco

* Department of Biomedical Engineering, Misr University for Science \& Technology, Cairo, Egypt

f Associate Professor, College of Nursing, University of Jordan, Amman, Jordan

9 eHealth Program Coordinator, Bamyan Provincial Hospital, Bamyan City, Afghanistan.

h Chief Medical Informatics Officer (CMIO) - SIDRA, Assistant Professor Clinical Pediatrics at Weill Cornell Medicine, Qatar

' Qatar Computing Research Institute, Hamad Bin Khalifa University, Doha, Qatar

i Faculty of Medicine, Ain Shams University, Cairo, Egypt

k Vice-Chair of Emirates Health Informatics Society, Head of eHealth Section at Dubai Health Authority, Dubai, United Arab Emirates

I Founder and Former Director of the Biomedical Informatics Center of Excellence (BMICoE), Information Technology Institute (ITI), Ministry of Communications and Information Technology (MCIT), Egypt

m President of Saudi Association for Health Informatics, Riyadh, Saudi Arabia

" Senior Consultant on eStrategies and eHealth to WHO and ITU, Geneva, Switzerland

- Department of Medicine, Weill Cornell Medical College in Qatar, Qatar Foundation, Doha, Qatar

p Executive Director of Nursing Informatics, Hamad Medical Corporation, Qatar

q Associate Professor of Nursing Management, Faculty of Nursing, Jordan University of Science and Technology, Irbid, Jordan

r Professor at Medical Informatics Laboratory, Faculty of Medicine and Pharmacy, Hassan II University, Casablanca, Morocco

\section{Summary}

There has been a growing interest in Health Informatics applications, research, and education within the Middle East and North African Region over the past twenty years. People of this region share similar cultural and religious values, primarily speak the Arabic language, and have similar health care related issues, which are in dire need of being addressed. Health Informatics efforts, organizations, and initiatives within the region have been largely under-represented within, but not ignored by, the International Medical Informatics Association (IMIA). Attempts to create bonds and collaboration between the different organizations of the region have remained scattered, and often, resulted in failure despite the fact that the need for a united health informatics collaborative within the region has never been more crucial than today. During the 2017 MEDINFO, held in Hangzhou, China, a new organization, the Middle East and North African Health Informatics Association (MENAHIA) was conceived as a regional non-governmental organization to promote and facilitate health informatics uptake within the region endorsing health informatics research and educational initiatives of the 22 countries represented within the region. This paper provides an overview of the collaboration and efforts to date in forming MENAHIA and displays the variety of initiatives that are already occurring within the MENAHIA region, which MENAHIA will help, endorse, support, share, and improve within the international forum of health informatics.

Keywords

eHealth, Middle East and North Africa, Health Informatics, collaboration, MENAHIA, Universal Health Coverage, Bioinformatics, International Medical Informatics Association 


\section{Introduction}

The goal of this paper is to convey to the IMIA community the importance of embracing the Middle East and North African Health Informatics Association (MENAHIA), which is being established as a regional non-governmental organization focusing on health informatics dissemination and uptake in the Middle East and North African Region. In the World Health Organization Eastern Mediterranean Region (WHO/EMR), health informatics is advancing in the application of information and communication technologies (ICTs) in health and biomedicine. Examples of that growth include the growing number of national eHealth or health informatics associations in the region including those in the Kingdom of Saudi Arabia, Islamic Republic of Iran, Kuwait, Lebanon, Morocco, Pakistan, Tunisia, and United Arab Emirates.

eHealth uptake in EMR countries has been supported by both the public and the private sectors as a means to enable equitable access to health services and information, to provide cost-effective solutions to the increasing burden of disease costs, and to contribute to Universal Health Coverage. It included development and deployment of electronic health records systems, electronic diseases monitoring, registration, and surveillance, health information on the Internet, and mobile health applications. Assisted by a fast-growing information and communications technology (ICT) penetration rate, especially via the Internet and mobile communications, many countries in the region have established academic programs for Biomedical and Health Informatics (BMHI) education as in Egypt and the Kingdom of Saudi Arabia or are in the process of doing so as in Morocco.

\section{The Need for a Regional Association}

Regional associations act as an umbrella organization for affiliated national associations. They emerge because of recognition by professional national bodies, institutions, and associations that they have common goals, which can be better and more effectively realized through collaboration and joint efforts. Regional organizations can provide an opportunity for networking and knowledge sharing, development of standards, and resource sharing and utilization. Every one of the national bodies realizes that working on its own will be costly and in most cases, wanted results may be impossible to achieve, making collaboration desirable and necessary. Examples of regional associations in eHealth exist in all continents of the world such as APAMI: Asia Pacific Association for Medical Informatics, EFMI: European Federation for Medical Informatics, HELINA: African Region, IMIA LAC: Regional Federation of Health Informatics for Latin America and the Caribbean, and IMIA North America. Of course the International Medical Informatics Association (IMIA)) being the home organization for all these regional bodies. Missing from the list of regions is the Middle East and North Africa, which include in alphabetical order: Afghanistan, Bahrain, Djibouti, Egypt, Iran, Iraq, Jordan, Kuwait, Lebanon, Libya, Morocco, Oman, Pakistan, Palestine, Qatar, Saudi Arabia, Somalia, Sudan, Syria, Tunisia, United Arab Emirates, and Yemen. These countries are the WHO member states in the EMR, which has its seat in Cairo, Egypt.

\section{Purpose of MENAHIA}

Founding of MENAHIA serves the goal to further the application of informatics methodology and information technology in the field of healthcare and biomedical research in the Middle East and North African region. The fundamental aims of MENAHIA as stipulated in its draft statute are to:

- Promote the health of people in the region and beyond through informatics in health care, public health, and biomedical research;

- Advance international cooperation of member organizations in the region;

- Stimulate research, development, implementation, and routine application of health informatics;

- Move informatics from theory into practice in the full range of health and care settings;

- Further the dissemination and exchange of knowledge, information, best practices, and technology;

- Promote education and training in health informatics;

- Support and advance accreditation of health informatics programs in the region to encourage a mobile work force in informatics;

- Promote the use and implementation of health informatics tools and technology in the Region;

- Promote responsible, professional, and ethical behavior of computer-based health data management;

- Seek and maintain formal channels of communication with any relevant professional or governmental organizations;

- Represent all member organizations in IMIA through an elected regional vice president for IMIA representing MENAHIA, and;

- Engage all members within the MENAHIA region through regional and international meetings and by calling periodic elections and ensuring representation from across the region on its governing board.

\section{Structure of MENAHIA}

MENAHIA is an association of regional health informatics associations. Modeled on the IMIA structure and other health informatics regional associations, membership in MENAHIA may besides include national organizations, academic and relevant industrial and trade organizations. MENAHIA will be open for corresponding members, who are individuals interested in joining and contributing to its objectives in countries without national associations.

\section{The Present Situation in the Middle East and North Africa}

To be able to understand the need for a strong regional organization to foster eHealth/ health informatics penetration, one has to understand the overall and specific conditions that impact eHealth development and uptake in the region. These include:

\section{Regional Digital Divide in eHealth}

While some countries in the Region enjoy a high penetration rate of ICTs including Internet and mobile telephony, many others fall within the very low penetration percentile; 


\section{eHealth Governance}

In most countries in the region, health information governance is very weak because of a lack of national strategies, plans, and policies or the non-adherence to those, where they exist. The result includes chaotic and fragmented health information technology development leading to costly or failed implementation;

\section{Public-private Partnerships}

Public-private partnerships are non-existent in many countries, which leaves the public sector isolated and in some countries as sole developer;

\section{Policy Development}

Engagement of the members of the public in policy development, monitoring, and accountability for health information technology is negligible, leaving citizens without advocates and with a lack of privacy protection and confidentiality and uncertainty in regards to data ownership;

\section{Standards and Interoperability}

Standardization and interoperability of systems are non-existent or lacking in some countries due to a lack of institutional/ governmental leadership and/or lack of a workforce with the required knowledge and skills;

\section{Language Barriers}

In the region, most eHealth resources and health information in electronic format are available predominantly in English. Language barriers provide obstacles in part due to mono-linguicism and domination of one language.

\section{Lack of Resources and Awareness}

Lack of regional and national resources and a lack of awareness of the proven benefits of health informatics in some countries, which diverts resources into more immediate health needs resulting in ineffective spending and a loss of sustainable and long-term benefits and savings that could have materialized.

\section{Lack of Education}

A lack of integrated health informatics education in university degrees results in a lack of critically needed trained graduates and health information technology professionals that may bring about or derive change.

\section{Weak National Health Information System (NHIS)}

All of the above challenges led to weak and/or fragmented NHIS in most countries. NHIS is considered the backbone for national Health System Strengthening (HSS) in order to realize the UHC goals - that aimed at providing quality-affordable health care services to everyone by 2030 [1].

The WHO report of 2016 " Global diffusion of eHealth: Making universal health coverage achievable" [2], noted that some progress had been made and emphasized that the existing health information technology gaps can be bridged through cross-fertilization and collaboration among countries. Currently, only twenty-two percent of EMR countries have developed policies or legislation to define medical jurisdiction, liability limits, and reimbursement of eHealth. However, $88 \%$ of the EMR countries provide eHealth pre-service training opportunities for health sciences students and professionals. While $87 \%$ of the EMR countries reported at least one type of mHealth program, $86 \%$ of the countries offer appointment reminders through $m$ Health applications and $79 \%$ of the countries use mHealth for community mobilization purposes. Yet, only $21 \%$ of the countries use mHealth for patient monitoring. The report showed that 14 EMR countries reported having telehealth programs. As much as $62 \%$ of the EMR countries reported using eLearning for education of college students, medical students, and physicians. In 70\% of the EMR countries, national legislation for protection of personal information and eHealth data exist, while $21 \%$ of the countries have national legislation to protect privacy of individuals' electronic health-related data. Unfortunately, only $8 \%$ of the EMR countries have legislation governing the sharing of health-related data. Countries of the EMR did not report any activities in legislation governing individual rights related to electronic health record data deletion, sharing, access, and correction.

\section{Highlights of eHealth Country Initiatives within the Eastern Mediterranean Region (EMR)}

There are a number of eHealth activities occurring within the Eastern Mediterranean Region countries. Highlights of these initiatives from different member countries are provided below. It is important to note that not all initiatives nor all countries are represented in the following summary. While the authors approached eHealth and health informatics leaders in the EMR, not all were able to contribute to this work. Key persons from Afghanistan, Egypt, Jordan, Morocco, Palestine, Qatar, Kingdom of Saudi Arabia, Sudan, Tunisia, and United Arab Emirates provided information. A summary of our findings are offered below:

\section{Afghanistan}

Afghanistan is renowned for its geographical remoteness, rugged terrain, harsh climate, and high poverty rate. Patients have to pay large sums for travel, food, and lodging just to journey from remote locations, where it is not possible to obtain high-quality diagnostic services and treatment, to higher level referral facilities making telemedicine of utmost importance. One of the most prominent eHealth initiatives in Afghanistan is being led by the Agha Khan Development Network (AKDN) eHealth Programme, which has been designed to address the challenge of providing Afghanis with access to healthcare services. The Programme leverages the power of ICT to link underserved communities with health care institutions and health care providers. The Programme also focuses on building the professional capacity for health care providers in Afghanistan. The AKDN eHealth Programme is unique in that it attempts to address three major barriers to healthcare - access, quality, and cost - in low-resource settings via ICT. 
eHealth services provided under the AKDN eHealth Programme include teleconsultations and eLearning sessions. Teleconsultations (live and store-and-forward) improve the health status of communities within defined catchment areas by delivering cost-effective diagnosis and treatment while reducing travel and other costs and time spent by patients and their families. eLearning sessions offered to health care providers under the Programme improve the skills and capacity of health care providers at the health care facilities.

\section{Egypt}

Since the early 80s Egypt's academic, public, and private sectors have paved the way to develop the biomedical health informatics (BMHI) discipline. In 2005, progress in developing the information society [3] was built on efforts from 1998 to 2000 by the Ministry of Communications and Information Technology (MCIT) to enable ICT in the health sector [4]. The national eHealth initiative - launched in early 2005 by MCIT, the Ministry of Health and Population (MoHP), and academic and private parties - supported eHealth projects in health administration, teleconsultation, and telemedicine [5].

Cairo University established the Systems and Biomedical Engineering Department offering a minor program in Biomedical Informatics. In 2004, the first Medical Informatics and Bioinformatics Master Program was developed by Helwan, Ain Shams, and Suez Canal Universities through a joint project funded by the European Commission (EC)-TEMPUS Program. Ain Shams University's Bioinformatics Department at the Faculty of Computer and Information Sciences is planning a Master's degree program in Health Informatics. Most private and international universities in Cairo offer bioinformatics courses. The Information Technology Institute (ITI) has adopted the IMIA-BMHI learning objectives for its capacity building programs in partnership with MoHP, which launched the first Egyptian health informatics fellowship program. Top Egyptian universities and ITI organize annual international conferences to address the new aspects of the BMHI discipline and its corresponding innovative technologies in healthcare [6].
In the private sector, the healthcare information industry burgeoned in early 80 s. International companies have implemented several eHealth projects in the Egyptian hospitals. Many companies and tech-startups compete in the digital health market providing applications for Health Information and Management Systems, Picture Archiving and Communications Systems, Pharmacy Management Systems, and mHealth [12].

In Egypt, a need remains to establish a national eHealth association as well as the legislation for eHealth services made more urgent, after the recent approval of the Universal Health Coverage (UHC) law to expand access to healthcare in Egypt [13].

\section{Jordan}

In 2009, Jordan established a not-for-profit company, called Electronic Health Solutions (EHS), that led the development, adaptation (from VistA), and deployment of an Open Source electronic health record system called Hakeem. Hakeem was the first national initiative of its kind to deploy ICT to an aspect of the public healthcare sector in Jordan. The program aimed to facilitate efficient, high-quality healthcare in the Kingdom through the nationwide implementation of an Electronic Health Record solution (EHR). In effect, physicians, pharmacists, medical technologists, and other clinicians in most of the government hospitals are at present able to electronically access medical records of patients within participating health facilities in Jordan simply by entering the patient's national ID number [14]. Nursing informatics has picked up in Jordan as nurses have started to use different electronic systems to document their notes and develop nursing care plans using computer-based systems. Most of the private hospitals have deployed solutions to serve both clinical and administrative/financial needs. Pharmacies in Jordan have computerized their inventories and tracking systems. Health informatics courses are being taught in a number of universities, but not leading to a degree yet. Efforts are being extended to establish and a national eHealth association in Jordan. Yet, despite these developments, little has been done to improve health information on the Internet in Jordan.

\section{Morocco}

A number of eHealth projects were conducted in Morocco over the past years. Most of the University Hospitals within the country have been implementing eHealth systems. These implementations improved patient care through drug management and medical appointment scheduling within the country. The main difficulty with eHealth implementation in Morocco includes health professionals' resistance to change. Morocco has also established collaborations with Europe to fund and disseminate research to reduce the digital healthcare divide within the country. The Ministry of Health recently piloted telemedicine initiatives within the country to improve access to healthcare services.

\section{Palestine}

In Palestine, funded through USAID, a project to reform and develop the health sector in Palestine was implemented from 2008 to 2013. The project was implemented as a joint collaboration with the Ministry of Health. In 2011, the project procured and implemented the first EHR system, named Avicenna [15] in its first government hospital as a pilot. Since then, Avicenna was implemented in nine governmental hospitals and its deployment is being expanded to private hospitals and clinics. The EHR system architecture was centralized to provide a distributed infrastructure making clinical patient records at different points of care available. Currently, the system contains 1.9 million patient records and supports other administrative healthcare functions. However, there are a number of patient record data quality issues that make a significant number of these records less useful than hoped. Quality issues are due to several factors both technical and procedural including poorly supported health professionals training resulting in faulty entry and capture of clinical data. Several private hospitals have either used Avicenna or developed their own custom EHR systems to support patient care functions, however, these systems lack interoperability and do not contribute to the central patient care record. A couple of initiatives on educational program development have also been 
started in Palestine, in joint collaboration with Jordan and some European countries, to bring health informatics training as part of mainstream university degree education [16]. Although the impact of these initiatives on the healthcare sector may be delayed, but many may be noticed earlier with the first waves of health informatics trained graduates entering the work force.

\section{Qatar}

The Qatar health service environment is composed of multiple provider organizations including government, private, and volunteer organizations. Government organizations include Hamad Medical Corporation (HMC) consisting of multiple general and specialist hospitals and Primary Health Care Corporation (PHCC) providing community care, including public schools clinics. Sidra Medicine is a dedicated hospital supported by Qatar Foundation that caters to women and children. These healthcare providers have adopted an electronic health record (EHR), which facilitates interdisciplinary care, communication, and adherence to recently developed clinical guidelines. In addition, patient portals are being rolled out. Many of the non-government healthcare organizations are also moving towards the shared EHR. In the future, the EHR will become the foundation for health informatics research in Qatar. A recent key initiative by the Qatar Ministry of Public Health is the National Diabetes Strategy to address diabetes, a significant health problem in Qatar and neighboring countries. As part of this strategy, a diabetes registry will be developed that will allow complete data collection for patients with diabetes across multiple healthcare sectors. This will provide a wealth of data for audit, clinical service evaluation, and research.

\section{Saudi Arabia}

Saudi Arabia has been experiencing a major transformation. Improving the healthcare services is a major component of Vision 2030 through enhancing the access to care, improved value of the provided care, and strengthening the preventive measures for the health status of the residents. Saudi
Arabia has a national health care system, in which governmental agencies provide $78.9 \%$ of total health services. A budget of SR 4 billion (US \$1.1 billion) has been allocated for this strategy to overcome the slow transition to IT and improve the quality of healthcare services. Although $40 \%$ of $\mathrm{MOH}$ hospitals currently have electronic health records, primary healthcare centers still rely on paper-based records and are in a transition to adopt information technology. Other governmental healthcare providers have followed suit and started adopting information technologies in their settings. The Ministry of National Guard- Health Affairs (MNGHA), Ministry of Defense Health Affairs, King Faisal Specialist Hospital and Research Centre, and Teaching Hospitals of Ministry of Higher Education may have less difficulty in incorporating such extensive change. Yet, the Saudi health system is facing a struggle in adopting health data standards. Within and across healthcare organizations, many different terminologies, messaging standards, content representations, and applications protocols are used as a means of carrying out care across multiple providers.

\section{Sudan}

Apart from several "Health Informatics" Training events, the Sudan experienced two major eHealth related achievements:

1) Sudan set up a National Strategy on eHealth in 2006, which was followed albeit not rigorously. Upon request, users may receive the document after re-authorization from the Federal Ministry of Health of Sudan. 2) The first National Telemedicine Network was established in 2007 linking four government general hospitals in four different regions ("Wilayat") with the University Hospital in Khartoum for Tele-radiology, Tele-pathology, and TeleSafePregnancy consultations. The same Telemedicine Network is also used to deliver and support major Tele-learning events.

\section{Tunisia}

On the eve of the reform of the health system in Tunisia, the modernization of the Health Information System was recog- nized as an essential component. The main priority programs of the Ministry of Public Health over the period 2016-2020 then 2020-2025 for development and support of digital health include:

- Finalize the deployment of the RNS-NG network to the front-line health facilities (2017-2020);

- Develop a strategy and action plan to modernize the Health Information Systems (2017);

- Upgrade the Hospital Information Systems (HIS) aims to improve patient care and minimize operating, administrative, and management costs;

- Develop and implement a medico-economic evaluation model of hospital services in 10 hospital sites;

- Deploy the Electronic Medical Record (EMR) in public hospitals to improve management and develop national registers for clinical and scientific research;

- Digitize the management and administration information systems in university and district hospitals and digitize prescriptions in regional hospitals;

- Secure and control the medication process from the national central pharmacy to the patient through digitalization, e-pharmacy, and daily and individual drug dispensing;

- Tele-radiology and digital archiving of radiology images (RIS/PACS) to overcome non-availability of radiology specialists in remote regions;

- Develop tele-services (tele-appointments, online directories, m-Cessation, m-Health, etc.);

- Develop territorial approaches to e-health and telemedicine experiences to support innovative telemedicine projects (tele-radiology, tele-consultation, telemonitoring, etc.);

- Support the Department of Pharmacy and the National Laboratory for Drug Control in their digitization strategy;

The Tunisian Society for eHealth and Telemedicine was established in September 2000 with the goal to advance the development of digital health and uniting the efforts by professionals in Tunisia and to further collaboration with relevant bodies abroad. 


\section{United Arab Emirates}

The United Arab Emirates (UAE) has greatly developed its healthcare system over the past forty years. Within the healthcare industry, there has been a strong investment into an eHealth infrastructure within the country. Applying the Electronic Medical Record Adoption Model (EMRAM), almost two-thirds (20/33) of hospitals are at Stage 2 and almost one-third (10/33) have reached at least stage 5. There are also a number of recent initiatives within the UAE such as the Riayati, which will unify medical records within the UAE and provide researchers with access to health data to conduct population health studies and provide patients with access to their own medical records. Lower costs by reducing duplicate healthcare services and improved healthcare surveillance are expected to improve the overall healthcare outcomes within the country. Recently, there has been a ban on handwritten prescriptions by clinicians, who are now required to complete electronic based pharmaceutical drug prescriptions. There is a strong eHealth movement within the UAE that is changing how healthcare is practiced within the country. Additional initiatives and partnerships are being conducted moving towards the complete digitization of healthcare.

\section{Conclusion}

MENAHIA is an emerging organization that will foster collaboration on health informatics research, education, and experiences among member countries. We anticipate that the newly developed MENAHIA mission and vision statement along with an inclusive, open, and collaborative organization will provide the needed impetus to push health informatics agenda within the MENA region. Although challenges in collaboration across such a large and wide geographical area are inevitable, MENAHIA will overcome them by being more engaged through consulting member organizations on initiatives conducted by MENAHIA. We anticipate that IMIA will also play a more significant and more progressive role in supporting collaborative efforts across the region.

\section{References}

1. Schmets G, Rajan D, Kadandale S. Strategizing national health in the 21st century: A handbook. World Health Organization, Geneva, 2016.WHO.

2. Global diffusion of eHealth: Making universal health coverage achievable: Report of the Third Global Survey on eHealth, Geneva: World Health organization; 2016.

3. United Nations. Economic and Social Commission for Western Asia (ESCWA). National Profile for the Information Society In Egypt. ESCWA; 2005. Available at: http://unpan1.un.org/intradoc/ groups/public/documents/unescwa/unpan030610.pdf. (Accessed: 26 March 2018).

4. ibid

5. MCIT. ICT for Health. Available at: http:// www.mcit.gov.eg/Digital_Government/ ICT_for_Health. (Accessed: 26 March 2018).

6. Hussein R, Khalifa A. Biomedical and Health Informatics Education and Research at the Information Technology Institute in Egypt. Yearb Med Infom 2011;6:161-8.

7. Hussein R, Winter A. Towards More Integrated Implementation of Healthcare Information Systems: Using the 3LGM2 for modeling the IHE-Scheduled Workflow Integration Profile, Proceedings of the 21st IEEE International Symposium on Computer-Based Medical Systems (CBMS), Jyväskylä, Finland, 2008. p. 650-2.

8. WHO. Global Observatory for eHealth. Available at: http://www.who.int/goe/policies/countries/egy/en/. (Accessed: 27 March 2018).
9. European Commission. A boost for health research and collaboration in Africa. Available at: https://ec.europa.eu/research/infocentre/ article_en.cfm?\&artid=37276\&caller $=$ FP. (Accessed: 27 March 2018).

10. The Egyptian Telemedicine Foundation website. Available at: http://telemedegypt.org/. (Accessed: 28 March 2018).

11. WHO EMRO. Health at your fingertips: Using mobiles to help diabetics in Egypt. Available at: http://www.emro.who.int/egy/egypt-news/ health-at-your-fingertips-using-mobiles-to-helpdiabetics-in-egypt.html. (Accessed: 26 March 2018).

12. MediFormatica. Healthcare IT in Egypt. Available at: http://mediformatica.com/index. php?option $=$ com_weblinks \& view $=$ category\&id=27:hospital-management-information-systems \&Itemid=103. (Accessed: 27 March 2018).

13. Devi S. Universal health coverage law approved in Egypt. Lancet 2018 Jan 20;391(10117):194.

14. Hakeem Program. Electronic Health Solutions. Available at: https://ehs.com.jo/hakeem-program. (Accessed: 5th March 2018) .

15. AviCenna HIS, DataSel. Available at http://www. datasel.com.tr/ (Accessed on 27 March 2018).

16. HiCure: Development of integrated health informatics curricula in undergraduate education. Available at http://sites.birzeit.edu/hicure/ (Accessed on 27 March 2018).

\section{Primary Corresponding Author}

Najeeb Al-Shorbaii

eHealth and Knowledge Management Consultant

Retired Director of Knowledge, Ethics and Research Department

WHO/HQ

Amman, Jordan

E-mail: shorbaiin@gmail.com

\section{Secondary Corresponding Author}

Dr. Mowafa Househ

Department of Health Informatics

College of Public Health and Health Informatics

King Saud Bin Abdulaziz University for Health Sciences

Ministry of National Guard-Health Affairs

Riyadh, Saudi Arabia

E-mail:mowafah@gmail.com 